\title{
Application of inclined injection piles as means of seismic protection of slope
}

\author{
Evgenii Kolosov ${ }^{1}$, Kamil Agishev ${ }^{2}$ and Natalia Kolosova ${ }^{1, *}$ \\ ${ }^{1}$ Peter the Great Saint-Petersburg Polytechnic University, Institute of Civil Engineering, \\ 195220 St. Petersburg, Russia \\ 2 TÜV International, 190000 St. Petersburg, Russia
}

\begin{abstract}
The using of inclined injection piles in an area with high seismic activity is described in this work with the purpose of their application in the construction of various facilities in seismic active areas. In this paper the authors define the possibility of their using for the stability of the slopes and slope construction in the earthquake conditions. The authors analyze sustainability slope both with the use of inclined injection piles and without them. They offer a methodology for determining the embedment of inclined injection piles into the thickness of slope.
\end{abstract}

\section{History of a pre-calculation of CFA piles in seismically active regions}

CFA piles - a kind of bored piles, the main distinguishing features are:

- small diameter - from 0.13 to $0.35 \mathrm{~m}$;

greater flexibility (with a length of piles over $10 \mathrm{~m}$ );

- used material (cement-sand mixture);

- process of molding of the concrete mix after filling the wells with the 0.2-0.4 MPa pressure.

In the domestic construction industry CFA piles have appeared in the middle of 1980s. They are mostly used to strengthen the foundations of buildings [1]. Currently, the range of use has significantly expanded; these piles use to protect against landslides, strengthening embankments, device enclosing walls in the ground, in the construction in dense urban areas and for other constructions [2-4].

The ability of the piles to resist both vertical and horizontal loads determined their application in seismically active areas $[1,5]$.

However, it should be noted that in the design of structures using CFA piles mainly used calculation methods for bored piles used in bridge construction. These piles compared to bored piles considerably differed by large diameter and their calculation methodology was quite complex and time-consuming $[5,6]$.

As shown by field tests of CFA piles the value of the bearing capacity of soil is much higher in various geotechnical conditions on the combined effect of vertical and horizontal

\footnotetext{
${ }^{*}$ Corresponding author: nb.kolosova@,yandex.ru
} 
forces [1]. Preliminary bearing capacity of piles at the construction site exceeded the bearing capacity of the pile calculated according to the procedures of the building regulations (excluding manufacturing technology piles), approximately 1.7 times. There were also significant differences in the determination of the incorporation of root anchor into the body of the slope [1].

The use of CFA piles as a stabilizing structure studied insufficiently in terms of earthquake. In this paper, the authors first conducted a comparative performance evaluation of CFA piles in normal conditions and under seismic activity.

As a design tool the authors use the software package PLAXIS 2D, which has demonstrated quite good results in the solution of various problems of soil mechanics [6,7].

\section{Calculation of inclined CFA piles with application PLAXIS 2D complex program}

\subsection{Calculation of slope stability in seismic conditions.}

The calculation of slope stability was carried out for two combinations of loads (including seismic component and without it). The cross section of the CFA pile is shown in Fig.1. Here is one of typical size roof bolt $40 / 18 \mathrm{~mm}$ and the CFA pile diameter equal to $150 \mathrm{~mm}$.

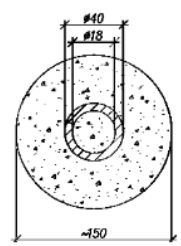

Fig. 1. The cross section of the CFA pile.

Mohr-Coulomb model was used as the estimated model. The stability calculation was performed based on the seismic forces that cause additional horizontal and vertical strains in the ground massif. And the role of vertical component of the seismic force was relatively small and was not taken into account when assessing sustainability.

Seismic force $\mathrm{Q}_{\mathrm{c}}$ in accordance with [8] is defined as the ratio of the weight of the soil compartment, which is undergoing a seismic impact:

$$
Q=\mu P
$$

$\mu$ - coefficient of dynamic seismicity (equal to 0.1 for areas with 9 points seismicity).

The direction of seismic force coincides with the direction of shear force in the corresponding compartment. As settlement scheme were used a real soil slope in one of the southern regions of the country exposed to seismic effects (Fig.2). The estimated seismic intensity was 9 points.

In the upper part of the slope was the retaining wall. The slope surface was represented by a broken line. The upper part value of the slope (CFA piles from №1 to №15) was $43^{\circ}$ in relation to the horizon. The lower part value of the slope (from №15 to №19) was $28^{\circ}$ in relation to the horizon. The height mark of the earth's surface was changing from +610.000 (pile №1) to +586.600 (pile №19). The total horizontal distance of the slope was 50.0 meters. 


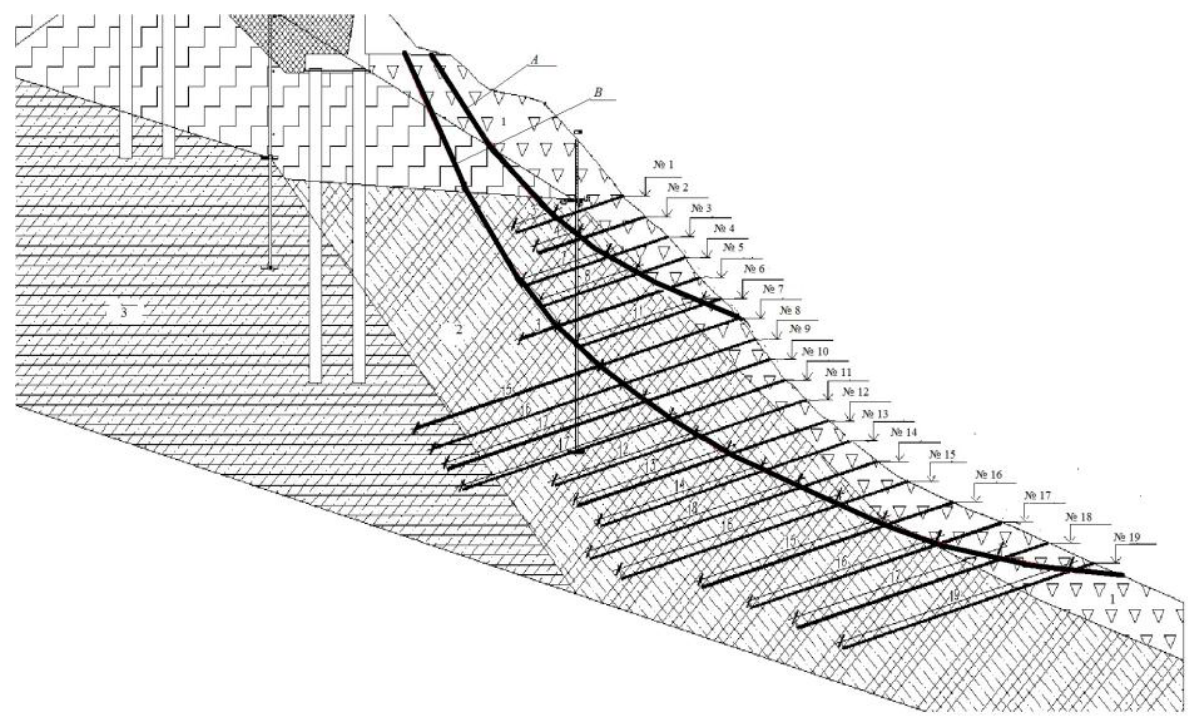

Fig. 2. The estimated scheme of the slope. $A$ - collapse curve without seismic load. $B$-collapse curve with taking into account seismic loads.

Composing the thickness of the slope soils represents three types: 1 - clayey medium fine gravel from $1.5 \mathrm{~m}$ to $4.0 \mathrm{~m} ; 2$ - clayey medium fine gravel fragments with low strength; 3 - claystone with very low strength. The main physical and mechanical characteristics are presented in Table 1.

Table 1. The main physical and mechanical characteristics of soil slope.

\begin{tabular}{|c|c|c|c|c|c|c|c|c|}
\hline \multirow[t]{3}{*}{ № } & \multirow[t]{3}{*}{ Name of soil } & 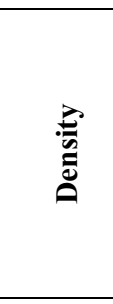 & 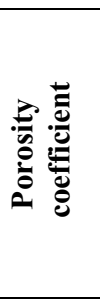 & 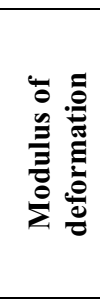 & 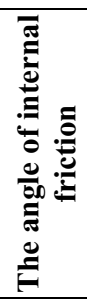 & 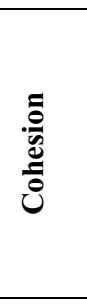 & 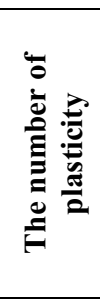 & 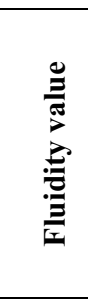 \\
\hline & & $\rho$ & $e$ & $\boldsymbol{E}$ & $\varphi_{1}$ & $c_{1}$ & $I_{p}$ & $I_{l}$ \\
\hline & & {$\left[\mathrm{kg} / \mathrm{M}^{3}\right]$} & & {$[\mathrm{MPa}]$} & {$\left[{ }^{\circ}\right]$} & {$[\mathrm{kPa}]$} & & \\
\hline 1 & Clayey medium fine gravel & 2070 & 0.609 & 29.5 & 23.9 & 12.9 & 0.123 & 0.173 \\
\hline 2 & $\begin{array}{l}\text { Clayey medium fine gravel } \\
\text { fragments with low strength }\end{array}$ & 2060 & 0.538 & 31.7 & 18.3 & 17 & 0.08 & 0.12 \\
\hline 3 & $\begin{array}{l}\text { Claystone with very low } \\
\text { strength }\end{array}$ & 2110 & 0.5 & - & - & - & 0.09 & $<0$ \\
\hline
\end{tabular}

Calculation of slope stability is determined in accordance with p.p.5.9 [8]. Estimated value of the soil mass of generalized resistance to the effects of shear should be determined based on the condition that the ratio between the normal $\sigma_{n}$ and tangential $\tau_{n}$ stresses across the sliding surface of the corresponding limit state sliding wedge meets the following condition 


$$
\tau_{n}=\sigma_{n} \operatorname{tg} \varphi_{1}+c_{1}
$$

$\varphi_{1}$ and $c_{1}$ - values of internal friction angle and specific cohesion of the soil in which occurs the shift of the soil.

The calculation was performed in the PLAXSIS 2D software package by reducing the strength characteristics of the soil. This process is called Phi-c reduction and present as a separate type of calculation. If use algorithm Phi-c reduction the strength parameters of the soil $\operatorname{tg} \varphi$ and $c$ were subsequently reduced until destruction of the structure.

The coefficient of slope's stability was determined as the ratio of the real resistance of the soil shear to the minimum shear strength required maintaining the balance.

According to the [8] for the structures of the normal level of responsibility the normalized slope stability factor is:

$K_{S}=1.15$ - at the basic load combinations,

$K_{S}=1.05$ - in special combinations of loads.

In our case, the basic mix includes a static state of the slope, taking into account the load on the road, and water saturation of the soil, and a special combination - seismic loads.

At first was determined a coefficient of slope stability for static condition without use of CFA piles. Its value amounted to $K_{S}=0.95$, which is less than the permissible values. This meant that the slope is not stable.

In the case of using CFA piles, the factor of stability for the basic load combinations amounted to $K_{S}=1.29$, and in special combinations $-K_{S}=1.1$. Realized calculation objectively demonstrated the feasibility of using CFA piles as strengthening of the structure during operation of the slope in seismic conditions.

\subsection{Calculation of bearing capacity and the depth of embedment of the injection anchor into the ground}

In addition to the calculation of slope stability was carried out the calculation for the determination of the anchor bearing capacity on the ground and the required value of the root sealing into the soil with the influence of seismic forces.

The calculation was performed for two load cases:

- without considering seismic forces (upper curve slide),

- with the seismic component (lower curve slide).

The bearing capacity of injection anchors $F_{d}$ is determined by the known formula:

$$
F_{d}=\pi D_{k} l_{k}\left(1+\sin \varphi_{1}\right) \cdot\left(\sigma_{s} \operatorname{tg} \varphi_{1}+c_{1}\right) K_{p} \gamma_{c},
$$

$D_{k}$ - the diameter of the anchor root sealing, $l_{k}$ - the length of the anchor root, $\varphi_{1}$ - the angle of internal friction of the soil, $c_{1}$ - specific adhesion of the soil, $\sigma_{s}$ - the stress of the ground by the side surface, $K_{p}$ - coefficient depend on the ratio of borehole diameter to the diameter of the sealing, $\gamma_{c}$ - the coefficient of working conditions.

The stress of the ground at the side surfaces $\sigma_{s}$ is determined depending on the specific weight of the soil subject to the weighing action of water $\gamma_{1}$, depth of the center of the seal anchor from the surface of soil coefficient of lateral earth pressure $\xi_{0}$ and the angle of the anchor to the horizontal $\alpha$. 
As initial values taken the following values: $\gamma_{1}=23.52 \mathrm{kN} / \mathrm{m}^{3} ; c_{1}=17.0 \mathrm{kPa} ; \varphi_{1}=18.3$; $\xi_{0}=0.55$; diameter of the root sealing $D_{k}=0.12 \mathrm{~m}$, the anchors angle to the horizon equal to $\alpha=15^{\circ}$ and $K_{p}=1.0 ; \gamma_{c}=0,9-$ the adopted coefficient of working conditions.

The value of embedment root of the injection anchor in the body of the slope was determined from the formula (3). The main results obtained in the calculation shown in Table 2 .

Table 2. Bearing capacity and drilling depth of injection anchor.

\begin{tabular}{|c|c|c|c|c|c|c|}
\hline 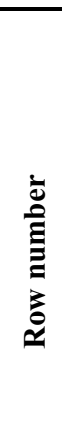 & 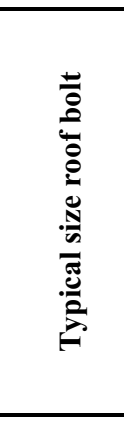 & 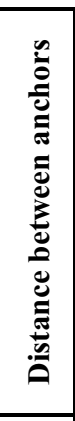 & 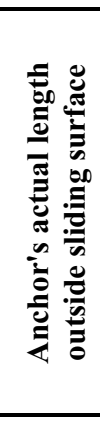 & 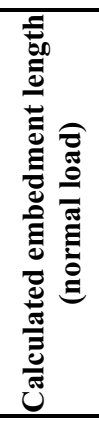 & 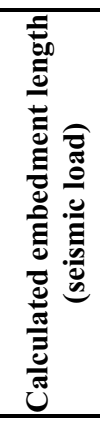 & 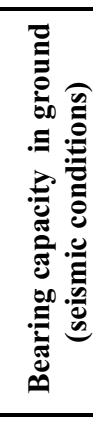 \\
\hline & $d$ & $\Delta l$ & $l_{k f}$ & $l_{k 1}$ & $l_{k 2}$ & $F_{d}$ \\
\hline & $\mathrm{mm} / \mathrm{mm}$ & $\mathrm{m}$ & $\mathrm{m}$ & $\mathrm{m}$ & $\mathrm{m}$ & $\mathrm{kN}$ \\
\hline 1 & $40 / 18$ & 2.0 & 3.00 & 2.00 & 8.10 & 83.3 \\
\hline 2 & $40 / 18$ & 2.0 & 4.00 & 1.00 & 8.10 & 110.7 \\
\hline 3 & $40 / 18$ & 2.0 & 7.00 & 4.00 & 8.70 & 203.8 \\
\hline 4 & $40 / 18$ & 2.0 & 8.00 & 6.00 & 8.80 & 235.2 \\
\hline 5 & $40 / 18$ & 2.0 & 12.00 & 5.00 & 8.80 & 352.8 \\
\hline 6 & $40 / 18$ & 2.0 & 11.00 & 4.00 & 14.70 & 488.0 \\
\hline 7 & $40 / 18$ & 1.5 & 15.00 & 14.00 & 6.70 & 361.6 \\
\hline 8 & $40 / 18$ & 1.5 & 16.00 & 5.00 & 19.53 & 906.5 \\
\hline 9 & $40 / 18$ & 1.5 & 17.00 & 5.00 & 20.00 & 982.9 \\
\hline 10 & $40 / 18$ & 1.5 & 17.00 & 5.00 & 21.00 & 1026.1 \\
\hline 11 & $40 / 18$ & 2.0 & 12.00 & 4.00 & 20.40 & 706.6 \\
\hline 12 & $40 / 19$ & 2.0 & 13.00 & 4.00 & 16.20 & 623.3 \\
\hline 13 & $40 / 20$ & 2.0 & 14.00 & 4.00 & 17.80 & 732.1 \\
\hline 14 & $40 / 21$ & 2.0 & 18.00 & 4.00 & 15.70 & 844.8 \\
\hline 15 & $40 / 22$ & 2.0 & 18.00 & 4.00 & 15.70 & 844.8 \\
\hline 16 & $40 / 23$ & 2.0 & 15.00 & 4.00 & 14.60 & 662.5 \\
\hline 17 & $40 / 24$ & 2.0 & 16.00 & 5.00 & 11.00 & 560.6 \\
\hline 18 & $40 / 25$ & 2.0 & 17.00 & 3.00 & 8.40 & 483.1 \\
\hline 19 & $40 / 26$ & 2.0 & 19.00 & 2.00 & 8.70 & 554.7 \\
\hline
\end{tabular}


The table shows data about actual embedment length for each anchor pile and calculated length under normal load and seismic component.

\section{Conclusion}

The first: calculation of slope stability using the software PLAXIS 2D showed that:

- The normative factor of slope stability was not provided below the retaining wall during the combined loads and seismic influence.

- To ensure the slope stability below the retaining wall proposed fixation of the slope by CFA piles with length from 9 to 27 meters with step $2 \times 2$ and $1.5 \times 2$ meters.

The second: comparing calculating data of the injection anchor sealing depth in the ground with the actual values of the embedment depth determined that:

- The actual length of each anchor embedment much higher (by 1.5 - 9.5 times) compared to the estimated for normal operating slope conditions.

- The value of anchor embedment into the ground is insufficient for piles № 1 - №4; №6; №8 - №13 in seismic conditions.

- The actual value of anchor embedment into the ground exceeds the calculated value for piles №5. №7, and №14 - №19 when working in seismic conditions. Exceeds 2 or more times for piles №7, №18 and №19.

In the conclusion should be noted the effective use of CFA piles as increasing slope stability elements with various combinations of load.

According to the author's opinion, CFA piles are possible use not only for strengthening the slope. Taking into account given calculation results, they can be used in other building structures.

\section{References}

1. V. N. Paramonov, Bearing capacity of piles in geotechnical conditions of SaintPetersburg. Proceedings of the International seminar on soil mechanics, 250-252, (2000)

2. A.S. Scalat, Evaluation of existing building in Israel for seismic hazard, Wiley Inter Science. Earthquake Engineering Dynamics 6, 3, 317- 325 (2007)

3. M. Pils, S. Parolai, M. Stupazzini, R. Paolucci \& J. Zschau, Modelling basin effects on earthquake ground motion in the Santiago de Chile basin by a spectral element code, Geophysical Journal International 187, 929-945 (2011)

4. G. Ya. Bulatov, N. B. Kolosova, E. S. Kolosov, A. B. Teplov, Dependence the bearing capacity of the pile on the shapes of cross-section, Construction of unique buildings and structures 10, (15),15-24 (2013)

5. V. V. Konyushkov, V. M. Ulitsky, Estimation of the bearing capacity of CFA piles, Bulletin of civil engineers 2, 52-57 (2007)

6. A. K. Bugrov, E. S. Kolosov, Practical methods of taking into account the nonlinear deformation of soils, IV International scientific-practical conference, COLL Actual problems of modern Foundation with energy saving technologies Penza, 14-21 (2014)

7. PLAXIS Software. https://www.plaxis.com/

8. SNiP 22-02-2003, Engineering protection of territories, buildings and structures against dangerous geological processes. Basic provisions (2003) 